\title{
L'AMÉNAGEMENT ÉNERGÉTIQUE DU DANUBE ALLEMAND
}

\author{
PAR
}

Sur le territoire de l'Allemagne Fédérale, le Danube se transforme progressivement à partir d'un petit ruisseau de moyenne montagne, jusqu'à devenir un fleuve écoulant, à l'aval de Passau, un débit en moyennes eaux comparable à celui du Rhin au droit de Ludwigshafen. La figure 2 montre les débits moyens du Danube et de ses affluents principaux, relevés sur une période de plusieurs années; on $y$ voit que les apports moyens annuels des affluents sont, dans deux cas (au confluent de l'lller à l'amont de la ville d'Ulm, et à celui de l'Inm à Passau) plus importants que le débil du Danube lui-mème.

La répartition des débits du Danube suivant les saisons est indiquée sur la figure 3, qui montre, pour différents endroits le long du cours, les débits moyens mensuels, relevés sur une période de plusieurs années, et comparés aux valeurs moyennes annuelles correspondantes. On reconnait, dans la partie supérieure du cours (limnimètre de Berg), la caractéristique d'un cours d'eau de moyenne monlagne, dont les débits importants s'écoulent surtout en hiver, avec une pointe tres nette au mois de mars. Le débit de l'Iller est un apport alpin qui, en augmentant les débits du Danube en été et en automne, compense la pointe printanière (limnimètre de Günzburg). Il en résulte une répartition très favorable suivant les saisons, et unique parmi les cours d'eau de l'Allemagne méridionale.

Plus loin à l'aval, et jusqu'à Passau, le Danube compte, le long de ses deux rives, de nombreux affuents dont la plupart arrivent du sud. Le Lech, de provenance alpine, présente des débits importants en été, mais ils sont compensés dans une certaine mesure par le réservoir de Forggensee, et

* Ingénieur diplómé à la Société par actions Rhin-MainDanube à Munich. par les apports du Wertach, à l'amont de son confluent avec le Danube. Les débits de l'Isar sont également plus réguliers que ceux des autres rivières alpines, grâce aux réservoirs de Walchensee et de Sylvenstein, aux lacs Starnberg et Ammer, et aux apports d'autres affluents. C'est ainsi que la répartition favorable des débits suivant les saisons est non seulement préservée dans les parties du cours du Danube plus à l'aval, mais même légèrement améliorée (limnimètre de Hofkirchen).

Le caractère du fleuve ne se modifie qu'à Passan, où l'Inn apporte un contingent où prédomine le caractère alpin, présentant une pointe au mois de juin, et un régime d'étiage pendant les mois d'hiver (centrale de Jochenstein). L'Inn présente également l'avantage que ses débits varient relativement peu d'une année à l'autre, parce qu'il est alimenté par des glaciers alpins. Par conséquent, le régime des débits du Danube allemand se prète mieux à la production hydro-électrique que celui de toul autre fleuve de l'Allemagne du Sud.

La pente du fleuve est, par contre, moins favorable. Le parcours, long de $167 \mathrm{~km}$, entre le limnimètre de Tuttlingen, dans le Jura souabe, et le confluent de l'lller, présente encore une pente moyenne de $1,06 \%$, mais celle-ci tombe à $0,8 \%$. entre Ulm et Ingolstadt $(128 \mathrm{~km})$, et à $0,46 \%$ entre Ingolstadt et Regensburg $(82 \mathrm{~km})$. La plus faible pente se situe sur le parcours, long de $119 \mathrm{~km}$, entre Regensburg et le limnimètre de Hofkirchen (à l'aval du confluent de l'Isar), où elle n'atteint plus que $0,21 \%$. Elle se raidit ensuite à $0,45 \%$ entre Hofkirchen et la frontière autrichienne à Jochenstein $(54 \mathrm{~km})$. C'est pour cette raison que l'exploitation systématique du cours n'a commencé que relativement tard. Avant 1950 , il ne fut construit que des centrales ou des groupes de centrales de faible puissance, en quelques endroits où les 
1/ Les centrales hydroelectriques

du Danube.

Die Kraftstufen an

der Donat.

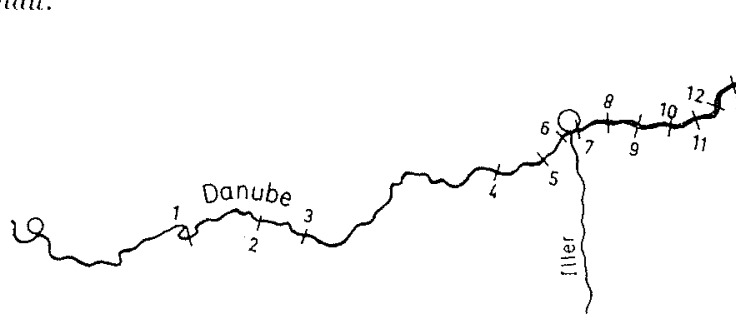

THBLEAU DES CENTRALES (TABELEE DER KRAFTSTUTEN)

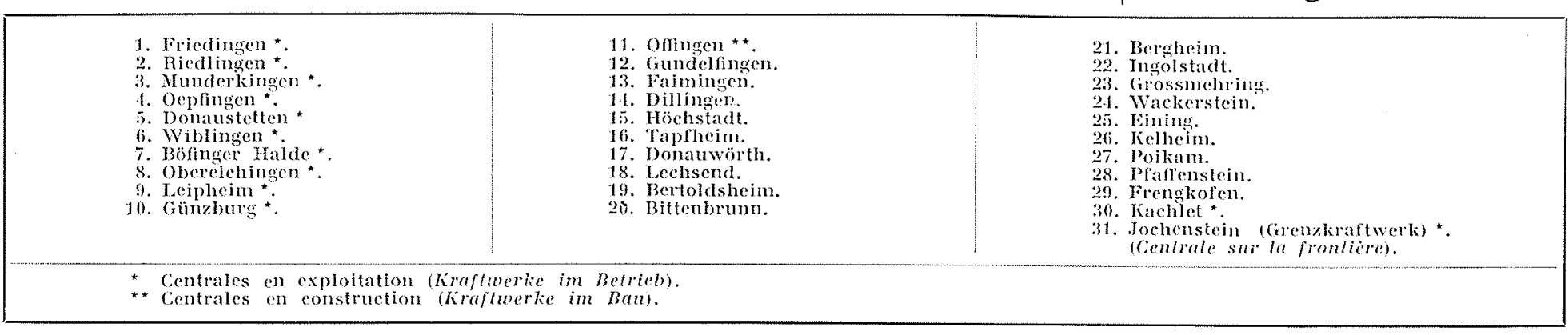

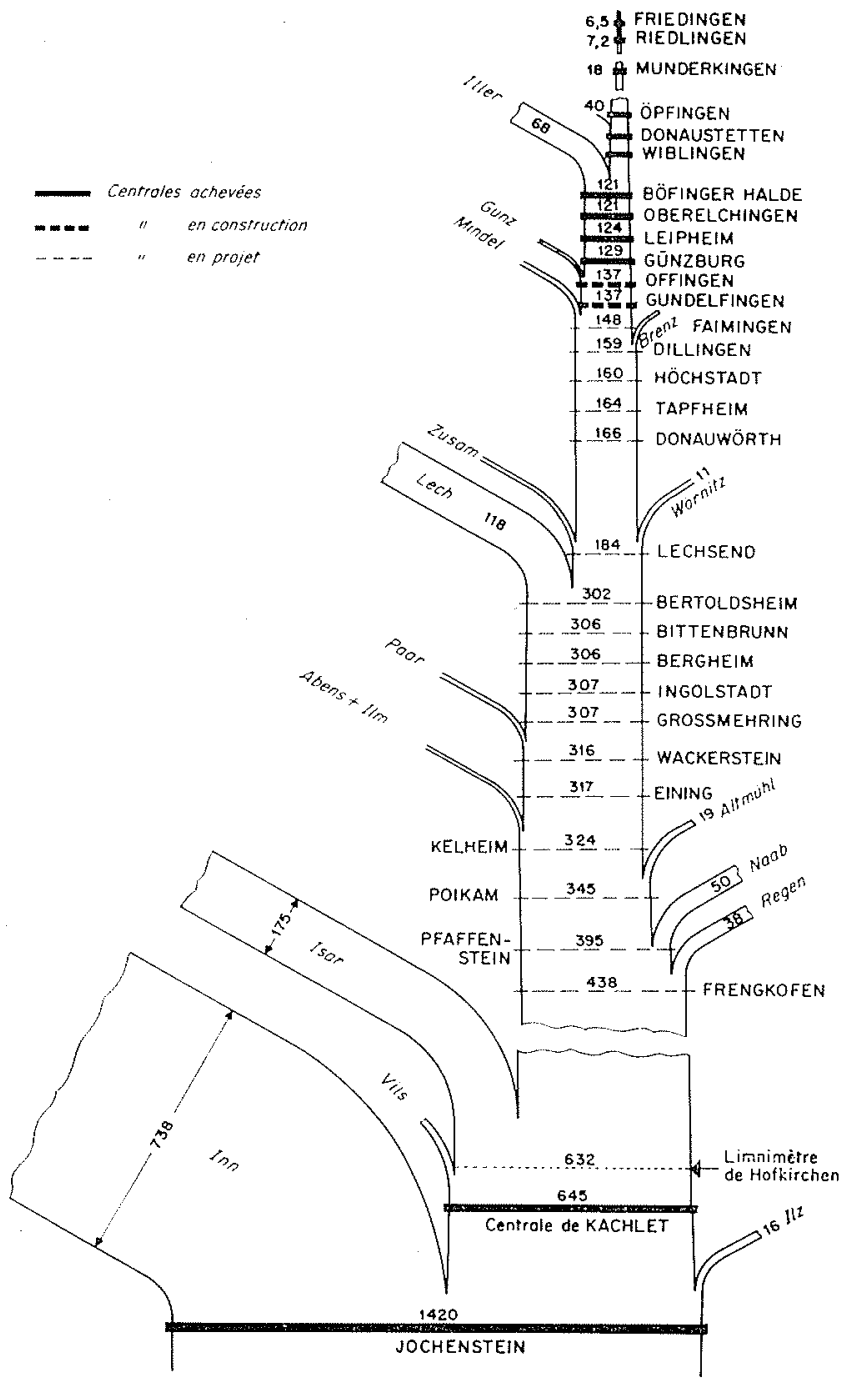

2) Les débits moyens du Danube allemand. Die mittleren Abfusswerte der Donau.

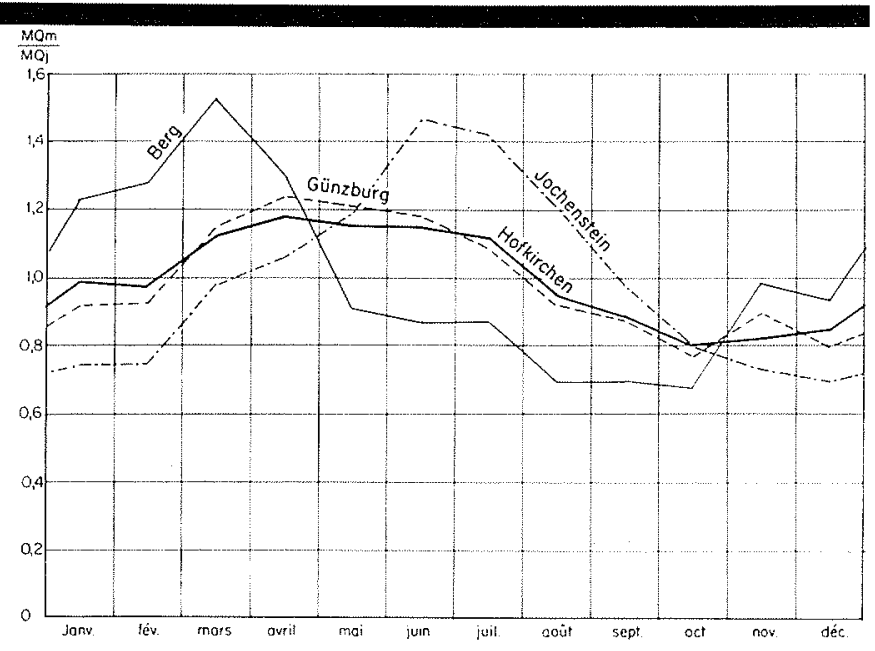

3/ Répartition saisonnière des débits du Danube. Jahreszeitliche Verteilung der Abflïsse.

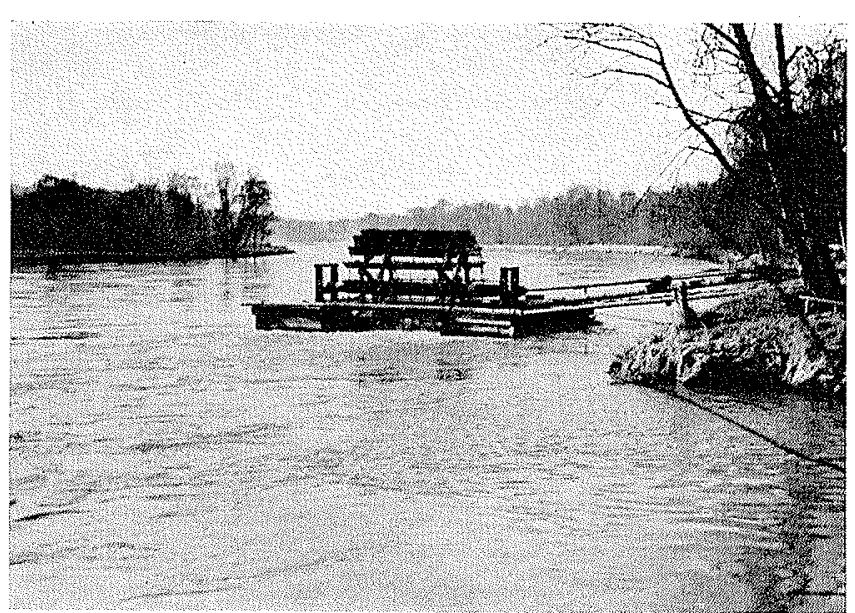

4/ Vieux hateau-moulin sur le Danube, près de Neuburg, en service jusqu'en 1942; au moyen d'un arbre a cardan, il actionna un moulin se trouvant sur la tere ferme, et plus tard un générateur électrique.

Alte Schiffsmïhle in der Donau bei Neuburg, in Betrieb bis 1942. Darch eine Kardanwelle wurde eine an Land befindliche Mühle, später ein Generator angetrieben. 
conditions locales rendaient judicieux de tels aménagements.

\section{Centrales à l'amont d'UIm.}

La première centrale équipée de turbines hydrau. liques fut construite en 1891 à Algershofen, à l'aval du confluent de la Lauchert. Elle a été remplacée en 1926 par la centrale de Munderkingen, présentant un débil équipé de $25 \mathrm{~m}^{3} / \mathrm{s}$, une chute nominale de $2,33 \mathrm{~m}$, une puissance de $0,433 \mathrm{MW}$, et une productivité moyenne annuelle de 2,7 GWh. Plus à l'amont, on a construit la centrale de Fridingen pendant: les années 1919 à 1923 (1,47 MW; $4,9 \mathrm{GWh}$ ) et celle de Riedlingen en 1925-1926 $(0,14 \mathrm{MW} ; 0,95 \mathrm{GWh})$. Ces trois centrales appartiennent aujourd'hui à la société «Energie-Versorgung Schwaben $A G »$, de Stutgart.

L'usine de Wiblingen a été mise en exploitation par la régie municipale de la ville d'Ulm en 1907 , c'est-à-dire avant l'achèvement du précédent groupe de trois centrales. Cette installation comporte quatre turbines Francis à axe vertical, entrainant quatre alternateurs triphasés par l'intermédiaire de trains de pignons coniques. La régie d'Ulm a aménage, par la suite, deux chutes complémentaires sur le parcours, long de $20 \mathrm{~km}$, à l'amont de Wiblingen : l'une à Oepfingen (en 1923), et l'autre à Donaustetten (en 1926). Les caractéristiques principales de ce groupe de centrales sont les suivantes:

\begin{tabular}{|c|c|c|c|c|}
\hline NoMr & 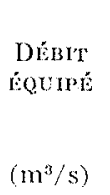 & $\begin{array}{l}\text { Chute } \\
\text { Eourbe } \\
\text { (m) }\end{array}$ & $\begin{array}{l}\text { Puissance } \\
\text { Jourbe } \\
\text { (MW) }\end{array}$ & $\begin{array}{l}\text { Product } \\
\text { VITE } \\
\text { ANNUELLE } \\
\text { MOYENNE } \\
\text { (GWh) }\end{array}$ \\
\hline $\begin{array}{l}\text { Öpfingen. . } \\
\text { Donaustetten. } \\
\text { Wiblingen. . }\end{array}$ & $\begin{array}{l}60 \\
80 \\
44\end{array}$ & $\begin{array}{l}5,5 \\
7,8 \\
3,7\end{array}$ & $\begin{array}{l}2,1 \\
4,5 \\
1,2\end{array}$ & $\begin{array}{r}8,2 \\
16,4 \\
6,4\end{array}$ \\
\hline Total. . . & & & 7,8 & 31,0 \\
\hline
\end{tabular}

Toutes ces centrales sont implantées sur des canaux latéraux comportant des réservoirs artificiels, et permettant le fonctionnement avec stockage quotidien. On envisage de remplacer dans quelques années l'usine de Wiblingen, devenue désuète, par une centrale en rivière dont le débil équipé sera du même ordre que celui de l'aménagement de Donaustetten.

\section{La centrale de Wachlet.}

Cette première grande centrale au fil de l'eau du Danube, siluée à $3,5 \mathrm{~km}$ à l'amont de la ville de Passau, a été réalisée à la même époque que le rroupe de centrales à l'amont d'Ulm.

Cet aménagement a été réalisé pour répondre au besoin d'assurer la navigation dans de bonnes conditions sur l'ensemble des $20 \mathrm{~km}$ du parcours rocheux et fissuré dit «du Kachlet»; il remplissait ce rôle en créant une retenue surélevant le plan d'eau d'environ $8 \mathrm{~m}$ et pouvant également servir à la production d'énergie hydroélectrique.

La société «Rhein-Main-Donau $A G »$, fondée en 1921, établit les plans d'exécution du projet et passa à l'exécution dans l'été 1922. Malgré des difficultés

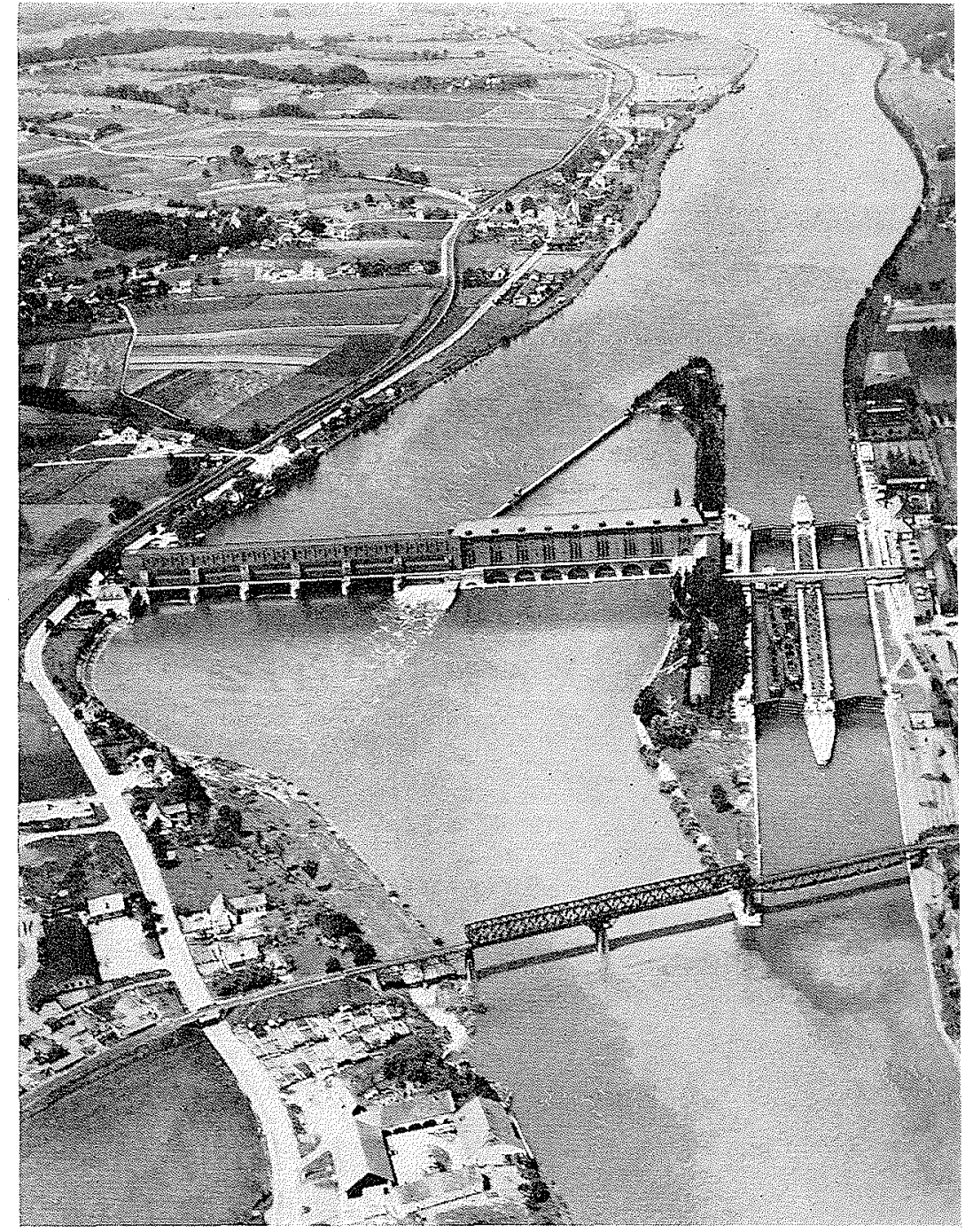

5/ Vue aérienne prise de l'aval de la centrale de Kachlet.

Das Kachletwerk, von der Unterwasserseite aus gesehen (Lufbild).

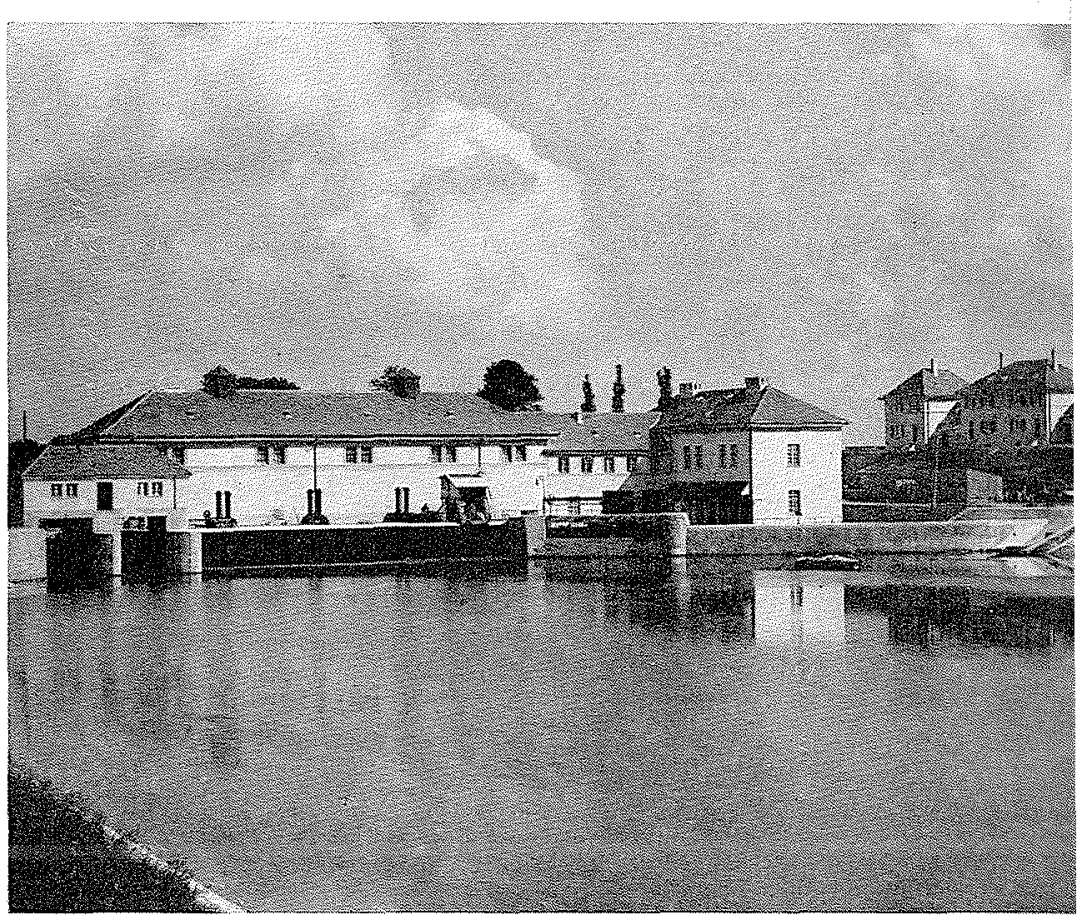

6/ Centrale de Donaustetten en amont d'Ulm.

Kraftwerk Donalistelten oberhalb von Ulm. 
dues aux dévaluations successives de la monmaie allemande à l'époque, les travaux purent être terminés dès le début de l'année 1928 .

L'ensemble de l'ouvrage présente une largeur de $220 \mathrm{~m}$ (fig. 6), et comporte six pertuis de barrags déversant, une usine avec huit groupes, et une ecluse double. Comme la turbine Kaplan se trouvait encore au tout premier stade de son évolution en 1922, le choix des groupes s'est porté sur huil turbines-hélice à axe vertical, chacune entrainant directement un alternateur triphasé. Cet ouvrage était à l'époque, et le resta encore longtemps après, une des plus grandes usines hydroélectriques d'Allemagne. Ses caractéristiques techniques étaient les suivantes :

Bassin versant............

Débit équipé.............

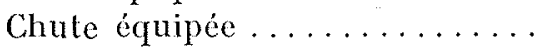

Puissance équipée...........

Productivité moyenne annuelle

(dont 48,5\% pendant les six

mois de l'hiver)...........

Diamètre des roues des turbines.

Vitesse ................

Diamètre des rotors des alterna-

teurs ................

Puissance unitaire des alterna-

teurs ................

Tension des alternateurs. . . . . .

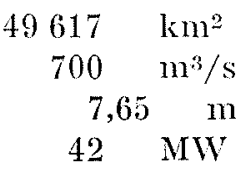

$\begin{array}{cl}262 & \mathrm{GWh} \\ 4,6 & \mathrm{~m} \\ 75 & \mathrm{tr} / \mathrm{mn}\end{array}$

$7,1 \mathrm{~m}$

$8,5 \quad$ MVA

$6,3 \mathrm{kV}$
Les possibilités d'une modernisation poussée de cette installation ont été étudiées lors des travaux de remise en état qui ont été jugés utiles après une période de fonctionnement de trente-trois ans $[10]$. On savait que la meilleure disposition pour les conditions données devait comporter seulement trois ou quatre groupes Kaplan de grandes dimensions, mais une telle modification aurait nécessité la transformation de l'ensemble de l'ourrage, ce qui aurait été extrêmement onéreux. Il se présentait, par contre, la possibilité de remplacer les roues des huit turbines-hélice par des roues à pales mobiles, installées dans les puits existants, et ainsi d'obtenir des turbines Kaplan avec un rendement et une capacité de débit plus élevés. Quelques modifications mécaniques complémentaires étaient également nécessaires, en particulier l'adjonction d'un élément d'arbre intermédiaire contenant le servomoteur de réglage des pales, et un nouveau rotor d'alternateur, à la fois adapté à la vitesse d'emballement plus élevée de la turbine Kaplan et améliorant le rendement de l'alternateur. Les ouvrages de génie civil pouraient, par contre, rester tels quels.

Les travaux de transformation ont commencé en 1961; on a prévu de remplacer les groupes deux à la fois, chaque changement étant effectué pendant la période d'étiage du fleuve au deuxiène semestre de l'année. Les résultats obtenus ont dépassé les prévisions du projet: à l'achèvement des travaux, en 1964, l'installation présentera un débit équipé de $1080 \mathrm{~m}^{3} / \mathrm{s}$, une puissance de $54 \mathrm{MW}$, et une productivité moyenne annuelle de $308 \mathrm{GWh}$, soit un accroissement de $18 \%$.

L'aménagement de Kachlet est resté pendant vingt-six ans le seul sur le Danube présentant une certaine importance, non seulement en Allemagne, mais également en Europe. Ce n’est qu'après la deuxième guerre mondiale que la décision fut prise de construire de nouvelles centrales hydroćlectriques sur ce fleuve.

\section{L'aménagement hydroélectríque de Jochenstein.}

A la reprise de ses activités de construction, la société «Rhein-Main-Donau AG » s'est occupée non seulement des aménagements hydroélectriques sur le Main, mais s'est également intéressée au projet d'une puissante centrale à Jochenstein en aval de l'embouchure de l'Imn, dont il avait déjà été question antérieurement. Au barrage même, et le long d'une grande partic de la retenue, la rive gauche du Danube est en Allemagne, et la rive droite en Autriche. A la suite d'un accord convenu avec la société «Osterreichische Elektrizitätswirtschaft $\mathrm{AG}$ (ÖV(x)», le projet défnitif a été mis au point par un bureau d'études conjoint. Par une convention signée en 1952 par les gouvernements de la République Fédérale Allemande, de l'Etal Libre de Bavière, et de la République Fédérale Autrichienne, fut fondée la société Donaukraftwerk Jochenstein $A G$, à Passau, à laquelle la OVG et la RMD participent chacune à raison de $50 \%$.

Un aspect intéressant de cet aménagement est le trace en plan de l'usine et du barrage, qui n'est pas rectiligne, mais légèrement incurvé, et convexe vers l'amont. avec un rayon de $1500 \mathrm{~m}$ (fig. 7). Cette disposition a été adoptée, parce que des essais sur modèle réduit à l'Institut Technique (Technische Hochschule) de Graz avaient montré qu'elle permettait d'assurer, pour le profil correspondant du lit du fleuve, les meilleures conditions d'écoulement à l'entrée des lurbines, et pour le passage des crues.

Le barrage comporte six pertuis équipés de vannes doubles cloisonnées à crochet pouvant évacuer, avec les écluses, un débit de crue exceptionnel de $11000 \mathrm{~m}^{*} / \mathrm{s}$. Le plus fort débit de crue enregistré jusqu'à présent (qui, d'ailleurs, s'est malheureusement produit pendant la construction des ouvrages en 1954) a atteint $9600 \mathrm{~m}^{3} / \mathrm{s}$.

La centrale, construite en forme de hall, comporte cinq groupes. Les écluses, de mêmes dimensions que celles de l'aménagement de Kachlel, s'y raccordent en rive gauche. La longueur de l'ensemble des ouvrages en travers du fleuve atteint $400 \mathrm{~m}$. Les caractéristiques techniques de l'aménagement sont les suivantes:

Superficie du bassin versant... $77025 \quad \mathrm{~km}^{2}$ Débit écuipé

Chute équipée.......... $8,75 \mathrm{~m}$

Puissance équipée......... 142 NW

Productivité moyeme annuelle. $940 \mathrm{GWh}$

Quantum de la productivité cor-

respondant aux six mois de

l'hiver...............

$44 \%$

Groupes et transformateurs :

- 5 turbines Kaplan, puissance unitaire $29 \mathrm{~kW}$, vitesse $65,5 \mathrm{tr} / \mathrm{mn}$, diamètre de roue $7,4 \mathrm{~m}$;

- 5 alternateurs triphasés, fournissant chacun $35 \mathrm{kVA}, 95 \mathrm{kV}$, diamètre du rotor $10 \mathrm{~m}$, excitation par ensembles de convertisseurs séparés: - 5 transformateurs de $35 \mathrm{kVA}, 9,5 / 220 \mathrm{kV}$ chacun, connectés en bloc aux alternateurs.

L'étroite collaboration entre l'Allemagne et l'Autriche, grâce à laquelle il a été possible de réaliser. cette centrale située à la frontière séparant les deux 


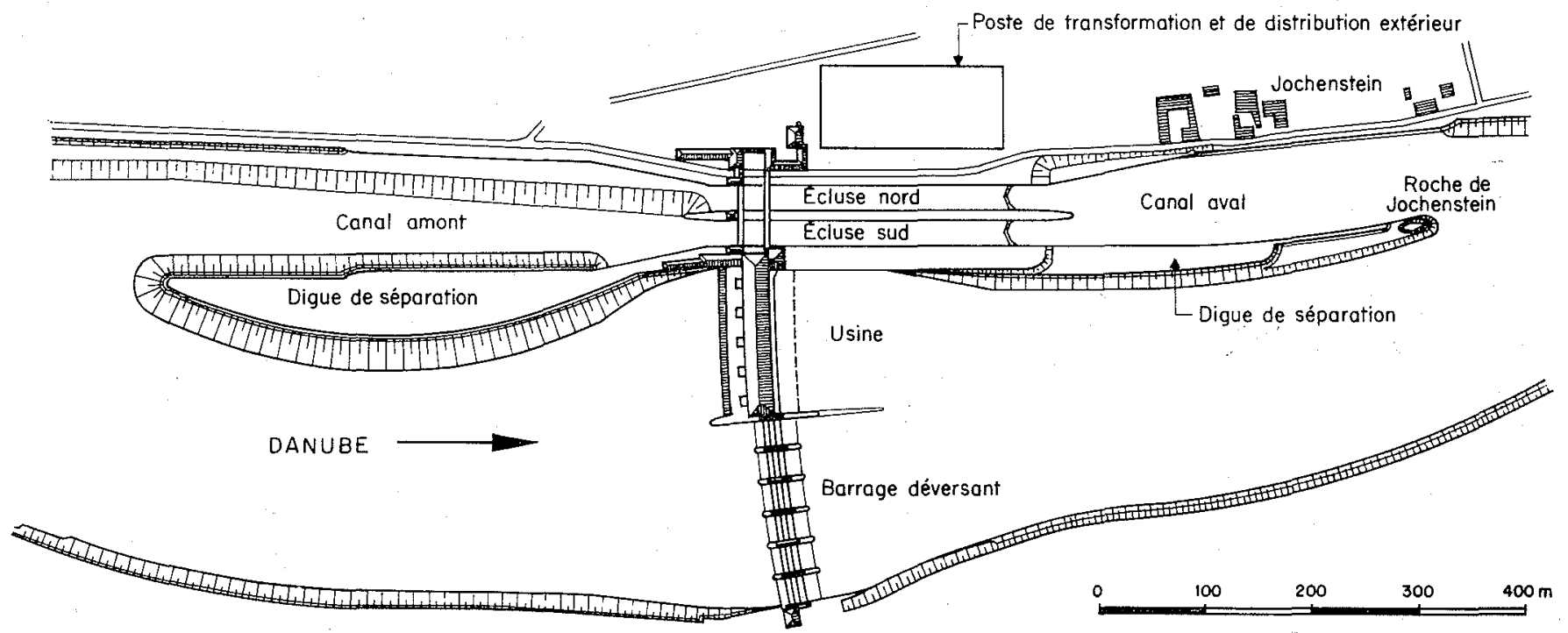

1/ Aménagement de Jochenstein, plan d'implantation.

Donaukraftwerk Jochenstein, Lageplan.

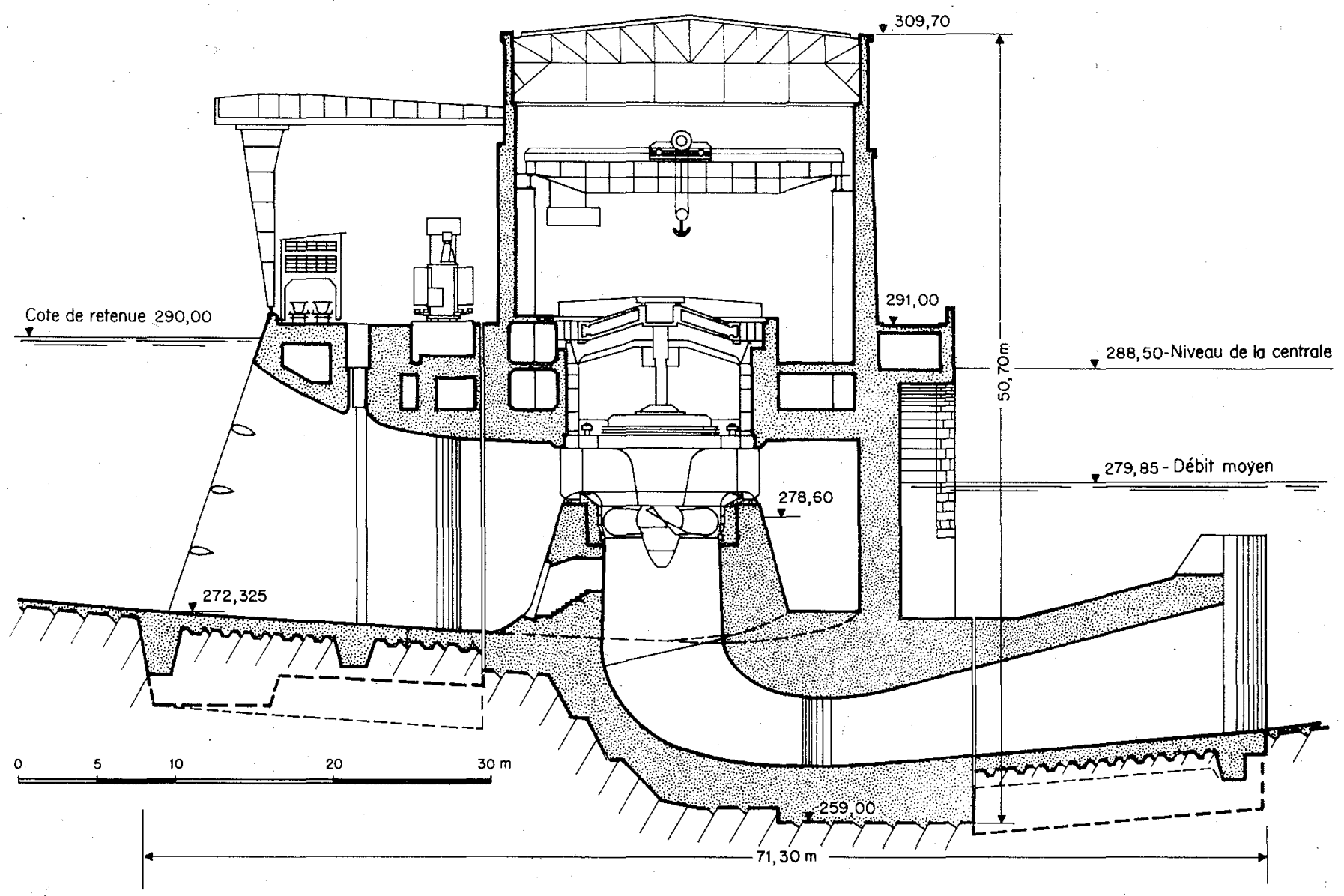

8/ Aménagement de Jochenstein, coupe verticale à travers la centrale.

Donaukraftwerk Jocheinstein, Schnitt durch das Maschinenhaus. 


\section{STEINHAUSER.}

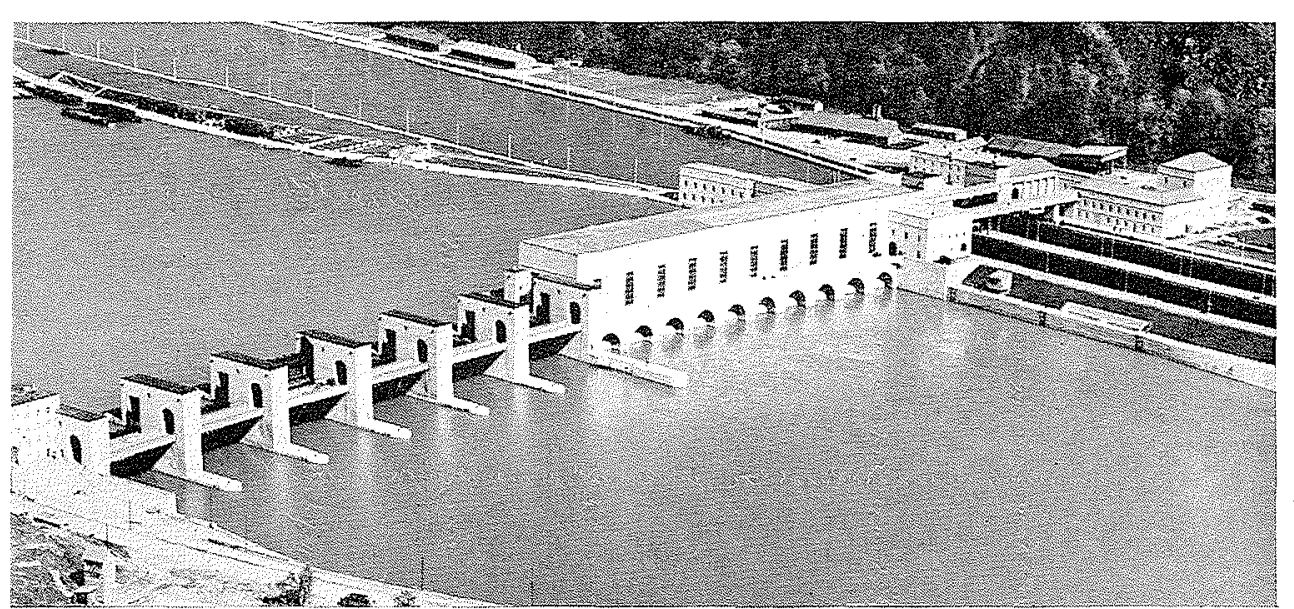

9/ Centrale de Jochenstein, vue aval prise de la rive autrichienne. Donaukraftwerk Jochenstein, Ansicht der Unterwasserseile vom österreichischen Ufer aus.

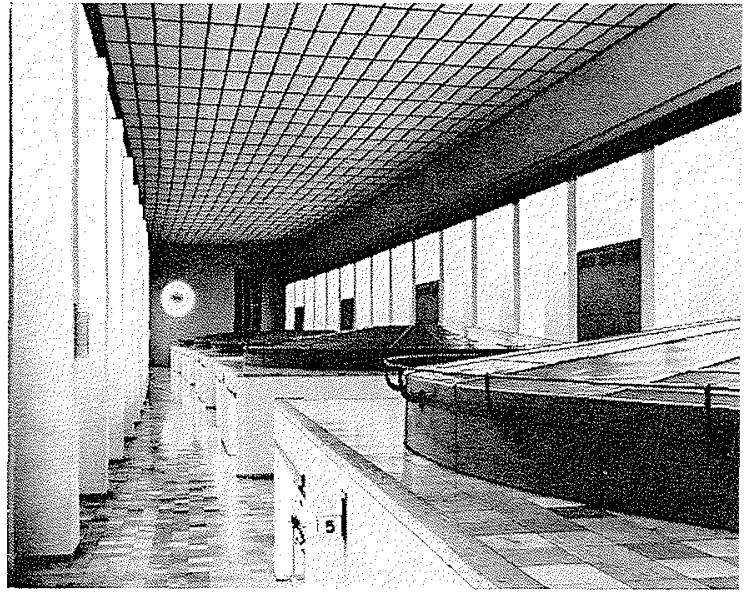

10/ Vue intéricure de la centrale de Jochenstein. Donalkraftwerk Jochenstein. Innenansicht. pays, a également conduit à une solution commune pratique du problème de la transmission de l'énergie produite. Une ligne double de $220 \mathrm{kV}$, d'environ $60 \mathrm{~km}$ de long, dont une moitie appartient à la Bayernwerk $\AA \mathrm{G}$, et l'autre à l'Oesterreichische Elektrizitätswirtschaft $\mathrm{AG}$, transporte cette énergie, en passant tantôt sur le territoire autrichien, tantôt sur le territoire allemand, jusqu'au poste de transformation de St.-Peter-am-Inn, près de la ville de Braunau, d'où elle est répartie entre l'Autriche et l'Allemagne.

Les premiers travaux de construction de l'aménagement de Jochenstein ont été entrepris pendant l'automne de l'année 1952. Les deux premiers groupes ont déjà pu être mis en service vingt-huit mois plus tard; le cinquième et dernier groupe est entré en fonctionnement le 20 aoùt 1956 , soit au bout d'une période de construction de quarantetrois mois seulement.

On envisage également l'aménagement d'une usine de pompage d'accumulation pour Jochenstein, pour laquelle la retenue servirait de bassin aval. Les caractéristiques géographiques du site sont très favorables pour un tel projet, car elles permettraient d'aménager, à $335 \mathrm{~m}$ au-dessus du plan d'eau de la retenue de Jochenstein, un bassin d'accumulation de 3,3 millions de mètres cubes, et représentant une énergie emmagasinée de quelque 2,3 millions de $\mathrm{kWh}$.

\section{W'aménagement hydroélectrique de Böfinger-Halde.}

La ville d'Ulm a entrepris pendant les années 1950-1952 (c'est-à-dire avant le début des travaux effectués à Jochenstein) la construction de la centrale de Böfinger-Halde à l'aval immédiat de la ville. Bien que cette centrale au fil de l'eau n'absorbe qu'un débit moyen de $121 \mathrm{~m}^{3} / \mathrm{s}$, et soit par conséquent beaucoup moins grande que celle de Jochenstein, elle a néanmoins représenté une nouvelle étape dans l'aménagement du Danube : celle

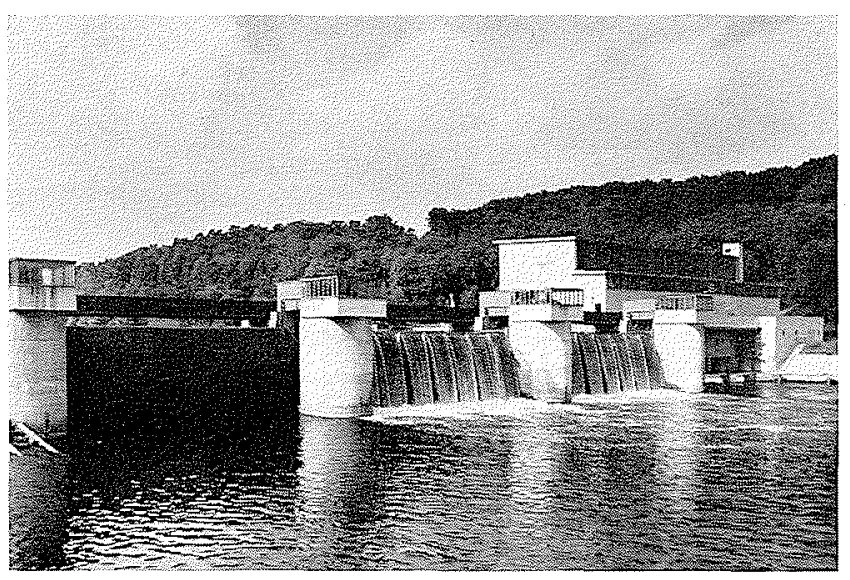

11/ Centrale de Böfinger Halde, près d'Ulm. Kraftwerk Böfinger Halde bei Ulm.

de l'aménagement systématique du parcours entre UIm et Kelheim, pour lequel est prévue une vingtaine de centrales en tout.

Le barrage, représenté sur la figure 11, comporte un déversoir à trois pertuis d'une largeur unitaire de $19 \mathrm{~m}$, une usine équipée de deux groupes Kaplan ì arbre vertical avec des alternateurs triphasés, les ensembles d'excitation étant montés sur les groupes. Les caractéristiques de l'aménagement sont les suivantes :

Superficie du bassin versant... $8040 \quad \mathrm{~km}^{2}$

Débit équipé........... $150 \mathrm{~m} 1 \mathrm{~m}^{3} / \mathrm{s}$

Chute équipée.......... $6,5 \mathrm{~m}$

Puissance équipée........ $7800 \quad$ kW

Productivité moyenne annuelle. 49 GWh

Diametre des roues de turbine. $3,89 \mathrm{~m}$

Vitesse .............. $107 \mathrm{tr} / \mathrm{mm}$

Puissance unitaire des alternateurs ............. 5,5 MVA

Tension des alternateurs .... $\quad 5,25 \mathrm{kV}$ 


\section{Centrales hydroélectriques de la Société "Obere Donau Kraftwerke AG ».}

La concession pour l'aménagement du cours du Danube allemand situé en territoire bavarois est détenue par la société "Rhein-Main-Donau AG». Cette concession intéresse donc également le parcours s'étendant entre la ville d'Ulm et Kelheim, bien que celui-ci ne soit qu'un troncon secondaire par rapport à la voie future «Rhin-Main-Danube ». Etant donné l'impossibilité de réaliser l'aménagement du fleuve en tant que voie navigable dans in délai prévisible, il a été décidé de n’équiper en première ítape que ses très intéressantes ressources hydroélectriques. Dans le but de faciliter le financement de ce projet, il a été créé, en 1958, d'un commun accord avec la société « Energieversorgung Schwaben $A G »$ et la Banque de Bavière (Bayerische Staatsbank), une filiale de la RMD, dénommée société "Obere Donau Kraftwerke AG», de Munich. Les activités de cette société n'intéres. sent cependant que six des dix-neuf centrales prévues entre les localités d'Oberelchingen et de Kelheim. Les premiers travaux de construction de l'usine d'Oberelchingen, située à $6,4 \mathrm{~km}$ à l'aval de celle de Ulm-Böfinger Halde (déjà mentionnée dans le présent texte), ont été entrepris en 1959. Elle a cté achevée en deux années à peine, et suivie à des intervalles d'un an par l'aménagement de Leipheim, de Günzburg, et d'offingen. Les centrales de Gundelfingen et de Faimingen, la dernière étape de la chaine, sont actuellement en cours de consIruction. Il est prévu de terminer l'ensemble de cette chaine de six centrales dès le printemps 1965.

Toutes ces centrales fonctionneront au fil de leau, et comprendront chacume deux groupes, dont les caractéristiques techniques sont résumées dans te tableau de la page suivante.

Ces centrales comportent chacune des déversoirs a trois pertuis équipés de vannes-segment surmontées de volets. Contrairement à la disposition classique, les pivots de ces vannes sont situés, non à l'aval, mais à l'amont, et au-dessous du plan d'eau. Par conséquent, les efforts de portée se transmeltent aux piliers sous forme de forces de compression, et non de traction, ce qui a permis de faire une économie appréciable sur les armatures nécessaires. Chaque groupe, à arbre vertical, comporte une turbine Kaplan entrainant un alternateur triphasé, l'excitation se faisant au moyen de redresseurs.

Les dimensions des retenues ont été choisies larges afin de permettre le fonctionnement en stockage quotidien.

\section{Aménagements ultérieurs.}

Après l'achèvemient de l'ensemble des centrales Oberelchingen-Faimingen en 1965, et des travaux ke modernisation de l'installation de Kachlet en 1965, la puissance installée du parcours allemand Au Danube s'élevera a environ $193 \mathrm{MW}$, et la productivité moyenne annuelle à environ $1165 \mathrm{GWh}$. Ces valeurs ne tiennent compte, pour Jochenstein, que de la participation allemande de $50 \%$. Parmi les quatorze étapes d'aménagements complémen-

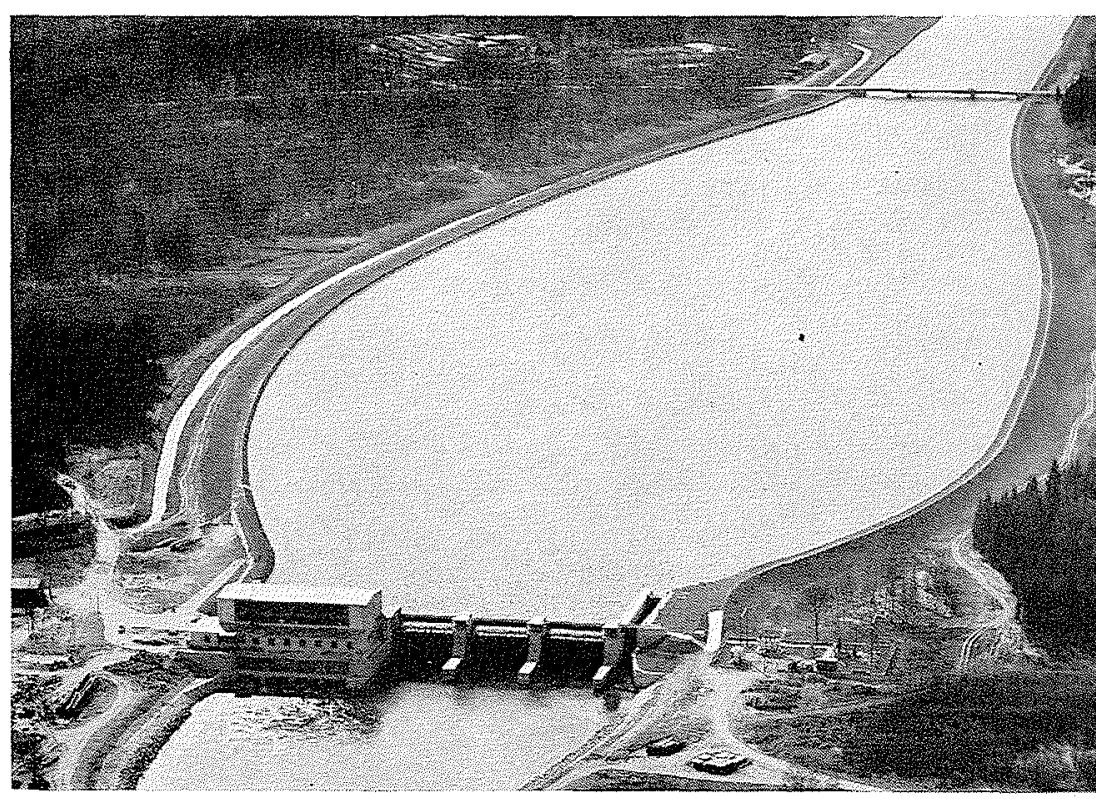

12/ Centrale d'Oberelchingen.

Donaulitaftwerk oberelchingen.

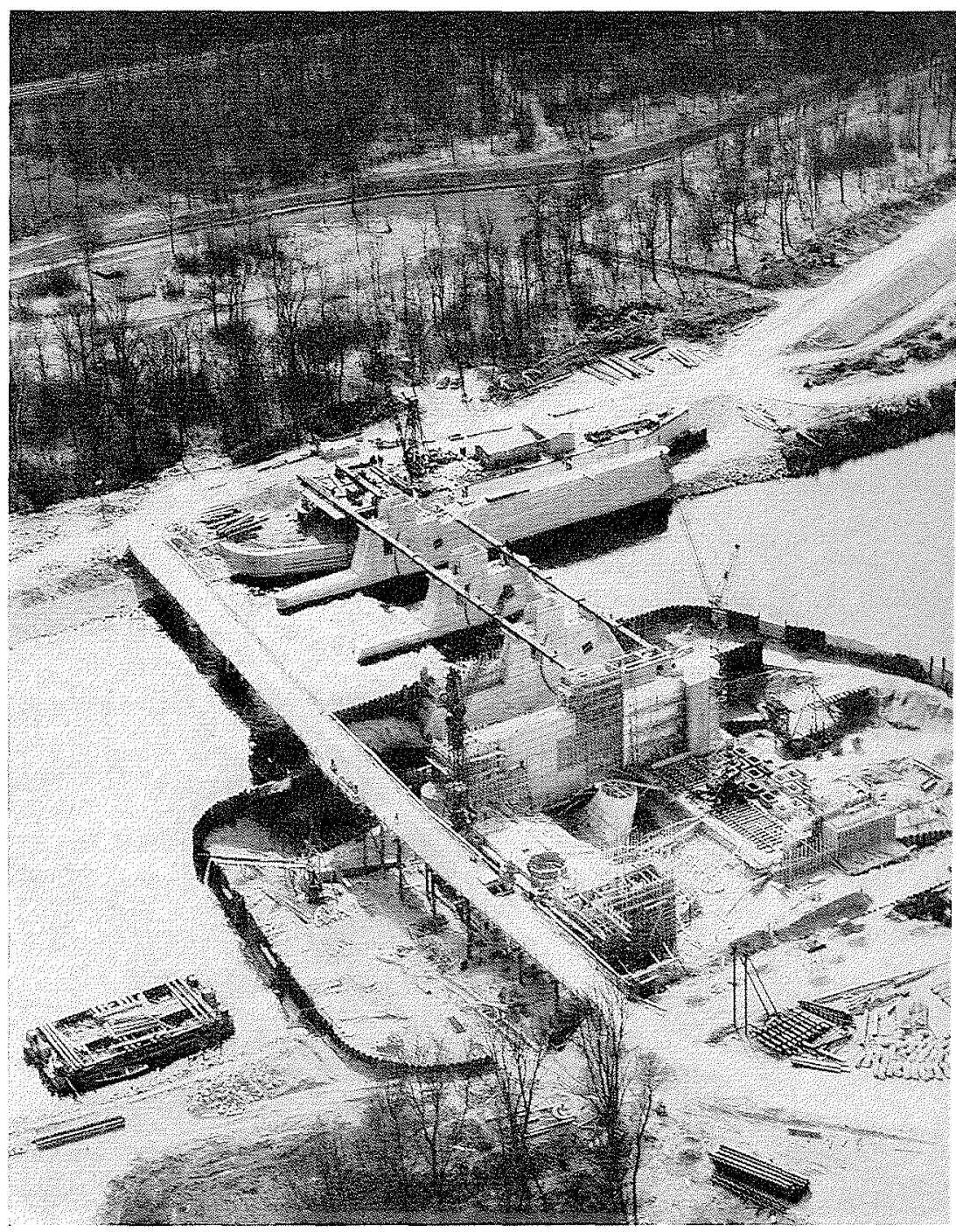

13/ Centrale de Leipheim. Vue du chantier en mars 1961 Donaukraftuerk Leipheim in Bau (Mär 1961). 


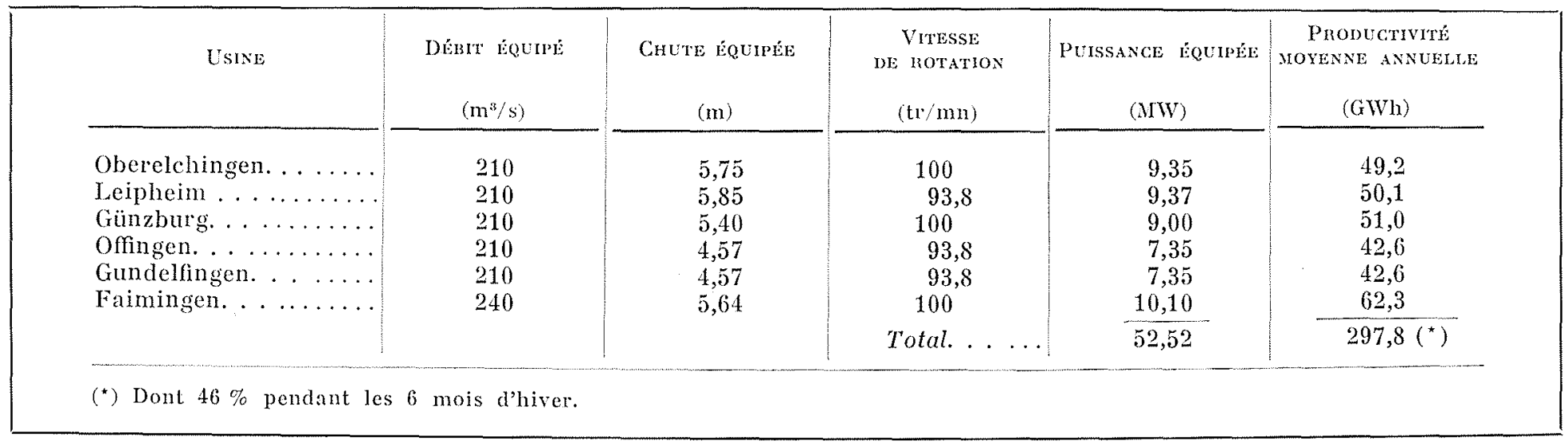

taires prévues dans la chaîne de centrales s'échelonnant d'Ulm à Kelheim, l'ensemble comprenant les centrales de Bertoldsheim; Bittenbrunn, Bergheim et Ingolstadt semblerait devoir être réalisé le premier. Son apport d'énergie s'élèvera à $75 \mathrm{MW}$, soit $480 \mathrm{GWh}$. On envisage également la construction de plusieurs retenues à l'aval de Kelheim, mais aucune décision n'a été prise quant à l'emplacement et la capacité de ces centrales hydro-électricques.

La capacité productive de l'ensemble du Danube allemand (à l'exclusion du parcours Frengkofen- barrage de Kachlet) est ćvaluée à $400 \mathrm{MW}$, soit une productivité moyenne annuelle de 2,5 milliards de kWh. Ces chiffres ne sont sans doute pas très impressionnants, si on les compare avec les possibilités qu'offre le Danube au-delà de la frontière allemande. Cependant, pour la République Fédérale Allemande, très appauvrie en ressources hydroélectriques non encore aménagées, le Danube présente un intérêt notable, surtout compte tenu de la répartition saisonnière favorable de sa productivité énergétique et des possibilités d'exploiter les aménagements en stockage quotidien.

\section{Bibliographie.}

[1] A. Kaspark. - Energiewirtschaft und Wasserstrassen. Elektrizitülswirtschaft, 1952, Heft 18.

[2] Kraftwerk Böfinger Halde, Ulm. Druchschrift der Stadtverwaltung, Ulm 1953.

[3] D) H. Fuchs. - Die Donaustaustufe Jochenstein. Die Wasservirtschaft, 1953, Heft 11/12.

[4] W. Lauz. -- Die Staustufe Jochenstein. Der Volkswirt, 1955 , Heft 43.

[5] Kachletwerk an der Donau. Druckschrift im Selbstverlag der $R M D), 1956$.

[6] Dr Böck. - Die Donaustauanlage Jochenstein. Die Wasseruirtschaft, 1957 , Heft 7 .

[7] Dr K. Fonrster. - Der heutige Stand und die mögliche Entwicklung des internationalen Donauverkehrs unter Berücksichtigung der Pläne für den energiewirtschaft- lichen Ausbau der Donau und für den Ausbau des Wasserstrassennetzes. Verlag Handelsblatt GmbH, Diisseldort, 1960

(8) P. Holders. - Ausbau der Oberen Donau und des Unteren Leehs durch die RMID. Denkschrift « Ausbat der Oberen Donau» des Verbandes Obere Donall e.V., Ulm 1961.

[9] L. Stennfauser. -- Kraftwerkskette an der Oberen Donav. VDI-Nachrichten, 1961, Nr. 26.

[10] F. Grermans und H. KuHs - Kinbau von Kaplanturbinen an Stelle von Propellerturbinen im Kraftwerk Kachlet der RMD. Elektrizitätsuirtschaft, 1962, Heft 6 .

(11) Dr. H. Fuchs, P. Holders und Dr. F. Schwalger. Wasserkraftnutzung an der Oberen Donau. Die Wasserwirtschaft, 1963, Heft 5 .

\section{Zusammenfassung}

\section{Der energiewirtschaftliche Ausbau der deutschen Donau Von Dipl. Ing;. L. Steinhauser *}

Die Wasserführung der deutschen Donaustrecke setzt sich aus Zuflüssen zusammen, die teils aus Mittelgebirgen, teils aus den Alpen stammen. Dadurch ergibt sich ein verhältnismässig gleichmässiges Dargebot, das für die ernergiewirtschaftliche Ausnutzung recht günstig ist. Weniger vorteilhaft sind dagegen die Gefälle, die auf dieser Strecke zur. Verfügung stehen.

Das erste grössere Flusskraftwerk an der Donal war das Kachletwerk, das 1922 bis 1928 im Zusammenhang mit der Schiffbarmachung der Strecke Passau - Regensburg arrichtet worden ist. Seine Ausbauleistung wird z.Zt. durch Umbau der Maschinensätze von 42 MW auf 54 MW erhöht. Nach dem 2. Weltkrieg wurde das deutsch -.. österreichische Grenzkraftwerk Jochenstein mit einer Ausbauleistung von $142 \mathrm{MW}$ errichtet. Etwa gleichzeitig begann die systematische energiewirtschaftliche Erschlissung der Donall unterhalb von Vlm. Dort sind 5 Verke fertiggestellt, 2 Werke im Bau, Die endgültige Zahl liegt noch nicht fest.

Im Ganzen können auf der deutschen Strecke (einschliesslich des deutschen Anteils am Jochensteinwerk) eine Ausbauleistung von $400 \mathrm{VW}$ und eine mittlere Jahreserzeugung von 2,5 Milliarden kWh erreicht werden.

\footnotetext{
* Dipl. Ing. in Fa. Rhein-Main-Donauaktiengesellschat, München.
} 